\title{
Análise da Variabilidade Genética Aditiva de Características de Crescimento na Raça Nelore ${ }^{1}$ \\ Roberta Lisboa Pontes Gestal de Siqueira², João Ademir de Oliveira ${ }^{3}$, Raysildo Barbosa Lôbo ${ }^{4}$, Luiz Antônio Framartino Bezerra ${ }^{5}$, Humberto Tonhati ${ }^{6}$
}

\begin{abstract}
RESUMO - Foram utilizados dados de cinqüenta e um rebanhos participantes do Programa de Melhoramento Genético da Raça Nelore (PMGRN), distribuídos nos estados de Goiás (GO), Mato Grosso do Sul (MS), Mato Grosso (MT), Minas Gerais (MG), São Paulo (SP), Maranhão (MA) e Bahia (BA). Foram obtidas estimativas de parâmetros genéticos para os pesos padronizados aos 120 (P120), 455 (P455) e 550 (P550) dias de idade. Análises unicaráter e bicaráter foram realizadas por modelo animal usando o aplicativo MTDFREML. Para P120 foi utilizado um modelo que incluiu como efeitos fixos, grupo de contemporâneos e classe de idade da vaca ao parto, e como aleatórios, os efeitos genéticos direto, materno e de ambiente permanente da vaca. Para P455 e P550, o modelo utilizado incluiu os mesmos efeitos fixos e o efeito genético direto do animal. Nas análises unicaráter, as estimativas de herdabilidade direta foram 0,29, 0,51 e 0,47 para P120, P455 e P550, respectivamente. Nas análises bicaráter, observaram-se coeficientes de herdabilidade direta de 0,50 e 0,58 para P120, 0,50 e 0,53 para P455 e 0,44 e 0,49 para P550. As correlações genéticas estimadas entre P120 e P455, P120 e P550 e P455 e P550, foram 0,92, 0,93 e 0,96, respectivamente. As estimativas de herdabilidade obtidas para P455 e as correlações genéticas deste peso com P120 e P550 sugerem que a avaliação genética pode ser feita aos 15 meses de idade em substituição aos 18 meses.
\end{abstract}

Palavras-chave: avaliação genética, bovinos, características de crescimento, Nelore, parâmetros genéticos

\section{Additive Genetic Variability Analysis in the Growth Characteristics of Nellore Breed}

\begin{abstract}
The data were obtained from 51 herds to participate in the Nelore Catttle Breeding Program (NCBP) from the states of Goiás (GO), Mato Grosso do Sul (MS), Mato Grosso (MT), Minas Gerais (MG), São Paulo (SP), Maranhão (MA) and Bahia (BA). Were used to estimative genetic parameters for standardized weights at 120 (P120), 455 (P455) and 550 (550) days of age. Univariate and bivariate analysis were performed by animal model using MTDFREML program. For P120 was used a model that included contemporary groups and cow age at calving as fixed effects, and direct genetic, maternal genetic and permanent environment effects as random effects. For P455 and P550 were utilized the same model but without maternal direct and permanent environment effects. The estimates of heritability direct from univariate analysis were: $0.29,0.51$ and 0.47 for P120, P455 and P550, respectively. In the bivariate analyses the direct heritability values were of high magnitude. The genetic correlation between P120 and P455, P120 and P550 and P455 and P550 were 0.92, 0.93 and 0.96, respectively. The values of the heritability coefficients estimated for the trait P455 and genetic correlation that characteristic with others indicate that the genetic evaluation could be made at the 15 months of age.
\end{abstract}

Key Words: bovine, genetic evaluation, genetic parameters, growth traits, Nellore

\section{Introdução}

A pecuária de corte no Brasil está experimentando importantes transformações, ressaltando-se a crescente busca dos produtores por tecnologias que possam resultar no incremento da rentabilidade do sistema de exploração. As fazendas estão se tornando empresas, contando com equipes de trabalho e assessorias nas áreas de melhoramento genético, nutrição, manejo e sistemas de criação, com introdução da informática no meio rural.

Até pouco tempo, o produtor de gado de corte além de abater os animais em idades tardias e com pesos elevados, ofertava carne de baixa qualidade ao mercado. Ademais, não percebia o custo que isso representava. Atualmente, a busca pela precocidade tornou-se uma prioridade tanto no meio rural como no científico. Este conceito tem sido muito discutido e

\footnotetext{
${ }^{1}$ Parte da dissertação de mestrado da $1^{\text {a }}$ autora apresentada à FCAV-UNESP, Jaboticabal.

2 Doutoranda, FMRP-USP/Professora e Coordenadora do Núcleo de Melhoramento Genético-UNIRP, São José do Rio Preto. E.mail: roberta@unirpnet.com.br

3 Departamento de Ciências Exatas-FCAV-UNESP, Jaboticabal. E.mail: jaoliv@fcav.unesp.br

${ }^{4}$ Professor Associado, Departamento de Genética e Matemática Aplicada à Biologia-FMRP-USP, Ribeirão Preto.

E.mail: rayblobo@genbov.fmrp.usp.br

${ }^{5}$ Analista de Sistemas, Departamento de Genética e Matemática Aplicada à Biologia-FMRP-USP, Ribeirão Preto.

E.mail: lafbezer@genbov.fmrp.usp.br

6 Departamento de Zootecnia - FCAV-UNESP, Jaboticabal. E.mail: tonhati@fcav.unesp.br
} 
vem gerando projetos de pesquisa com o objetivo de identificar critérios de seleção para obtenção de animais precoces, favorecendo a rentabilidade do sistema, já que reduz o tempo de permanência dos animais nas fazendas, além de colocar um produto final de melhor qualidade para o mercado consumidor.

A redução na idade da pesagem dos animais participantes de programas de melhoramento genético e, conseqüentemente, no número de pesagens é uma proposta de grande importância, pois permite aos criadores comercialização antecipada dos machos e seleção das novilhas para entrarem em reprodução a menor idade.

A reprodução em gado de corte é de fundamental importância econômica, portanto, a precocidade sexual irá interferir no desempenho lucrativo do sistema de produção.

As estações de acasalamento estabelecidas pela maioria dos criadores do PMGRN vão de outubro a março, no entanto, estão sendo atrasadas para o início de dezembro, com o objetivo de aproveitar a época de melhor qualidade das pastagens. Dessa forma, os animais atingem os 15 meses de idade no meio do período das águas quando estão ganhando peso, podendo, então, serem comercializados antes da época seca, com bom estado corporal e sem custos de suplementação.

Em melhoramento animal, o conhecimento de estimativas de parâmetros genéticos de características de importância econômica é o primeiro pré requisito para o estabelecimento de um programa de seleção. Na literatura, Eler et al. (1996), Lôbo et al. (1997), Reyes et al. (1997) e Garnero et al. (1998), em rebanhos da raça Nelore, obtiveram para o peso aos 120 dias de idade, estimativas do coeficiente de herdabilidade direta, variando de 0,20 a 0,27 , e para a materna, variando de 0,16 a 0,31 . Para o peso aos 550 dias de idade, as estimativas da herdabilidade direta variaram de 0,19 a 0,35 . Com relação às correlações genéticas entre P120 e P550, as estimativas variaram de 0,68 a 0,78 . Quanto ao peso aos 455 dias de idade, não foram encontradas referências na literatura sobre este parâmetro.

Assim, o objetivo do presente estudo foi estimar parâmetros genéticos para as características de crescimento, pesos padronizados aos 120,455 e 550 dias de idade (P120, P455 e P550), incluídas na avaliação genética do Programa de Melhoramento Genético da Raça Nelore, visando uma proposta para redução de pesagens e na idade das mesmas.

\section{Material e Métodos}

Foram utilizados dados de 51 rebanhos Nelore oriundos de fazendas participantes do Programa de Melhoramento Genético da Raça Nelore, localizadas nos Estados de Goiás, Mato Grosso do Sul, Mato Grosso, Minas Gerais, São Paulo, Maranhão e Bahia

Todas as fazendas adotavam estação de acasalamento que variava entre 60 e 90 dias de duração, sendo que algumas possuíam duas estações conforme a região e o ano. Geralmente, utilizava-se a inseminação artificial como técnica para reprodução dos animais, podendo ter repasse das fêmeas com touros, ou adotar-se exclusivamente monta natural. A desmama dos animais ocorria entre 7 e 8 meses de idade; a vacinação e os demais cuidados sanitários eram feitos conforme normas estabelecidas pelos órgãos de controle sanitário de cada região. Os rebanhos passavam por controle periódico e criterioso das características produtivas e reprodutivas, pois realizavam pesagens dos machos e das fêmeas de três em três meses (janeiro-abril-julho-outubro), do nascimento até 21 meses de idade e na ocasião da desmama, vaca e bezerro. A cada pesagem, a situação dos animais (normal, doente..) era informada. No cadastro, além da identificação do animal e dos pais, datas de cobertura e ou inseminação artificial, data e ocorrência no parto (normal, ajuda, cesárea) e a situação ao nascer (normal, natimorto..) também eram informadas.

As características estudadas foram os pesos padronizados para 120 (P120), 455 (P455) e 550 (P550) dias de idade. O ajuste dos pesos para as idadespadrão foi feito por interpolação linear.

Os grupos de contemporâneos para as características estudadas foram constituídos por animais nascidos na mesma fazenda, do mesmo sexo, na mesma época e mesmo ano e no mesmo regime alimentar.

Para P120, foram consideradas quatro épocas na formação dos grupos de contemporâneos, ou seja, $1=$ janeiro a março, $2=$ abril a junho, $3=$ julho $a$ setembro e 4 = outubro a dezembro; e para P455 e P550, duas épocas: 1 = outubro a março e $2=$ abril a setembro.

A razão pela qual se consideraram apenas duas épocas para P455 e P550 foi a diminuição no número de animais nos grupos de contemporâneos quando se consideraram quatro épocas, o que acarretaria diminuição no valor estimado da acurácia. 
A condição do regime alimentar foi estabelecida de modo a combinar todas as formas distintas de alimentação recebidas pelo animal durante toda sua vida, em cada idade-padrão analisada. Assim, um animal que, por exemplo, permaneceu em pasto do nascimento até o desmame e depois passou para regime de confinamento e a característica de interesse é o P455, seu regime alimentar (ra) foi codificado como 1133 ( 1 = pasto e 3 = confinamento), sendo que para P120, ra=1; para P240 (desmame), ra $=11 \mathrm{e}$ para P365, ra $=113$. Ou seja, todos os manejos adotados foram considerados em cada idade padrão analisada.

A idade da vaca ao parto (CIVP) foi agrupada em seis classes: menor que 36, de 36 a 47, de 48 a 59, de 60 a 71 , de 72 a 119 , e maior que 120 meses de idade

Consta na Tabela 1 o número de observações utilizadas nas análises para cada característica, bem como o número de animais na matriz de parentesco, o número de grupos de contemporâneos e o número de classes de idade da vaca ao parto.

As análises para estimação dos parâmetros genéticos foram efetuadas pelo método de Máxima Verossimilhança Restrita (REML) utilizando-se o software MTDFREML (Multiple Trait Derivative Free Restricted Maximum Likelihood), apresentado por Boldman et al. (1993). Para tal, foram fornecidos valores iniciais, previamente calculados, dos componentes de variância e, a cada interação, novos valores foram calculados até que a função fosse maximizada, quando então a convergência era atingida (com valores da função $-2 \log \mathrm{L}$ inferiores a $10^{-9}$ ). A cada convergência, o programa foi reiniciado, usando como valores de (co)variâncias iniciais aqueles obtidos na análise anterior, checando, assim, possíveis máximos locais e assegurando que o valor alcançado fosse o

Tabela 1 - Número de observações $(N)$, número de animais na matriz de parentesco $\left(\mathrm{A}^{-1}\right)$, número de grupos contemporâneos (GC), número de classes de idade da vaca ao parto (CIVP) das características estudadas

Table 1 - Number of records $(N)$, number of animals in $A^{-1}$, number of contemporary groups (GC), number of dam at calving classes (CIVP) for studied characteristics

\begin{tabular}{lcccc}
\hline $\begin{array}{l}\text { Características } \\
\text { Characteristics }\end{array}$ & $\mathrm{N}$ & $\mathrm{A}^{-1}$ & G.C. & CIVP \\
\hline P120 & 7.142 & 12.662 & 173 & 06 \\
P455 & 7.142 & 12.662 & 111 & 06 \\
P550 & 6.999 & 13.014 & 147 & 06 \\
\hline
\end{tabular}

\footnotetext{
R. Bras. Zootec., v.32, n.1, p.99-105, 2003
}

máximo global. Este procedimento foi repetido entre 2 e 5 vezes, segundo as características estudadas, até que os componentes estimados não diferissem entre uma análise e outra.

A princípio, cada característica foi analisada individualmente. Para o peso aos 120 dias de idade, foi utilizado um modelo estatístico que incluiu como efeitos fixos o grupo contemporâneo e a classe de idade da vaca ao parto e, como aleatórios, os efeitos genéticos direto, materno e de ambiente permanente da vaca.

Para as características P455 e P550, foi utilizado um modelo que incluiu os mesmos efeitos do anteriormente explicitado, exceto os efeitos aleatórios genéticos materno e de ambiente permanente da vaca, devido ter sido verificado em análises prévias que a contribuição destes efeitos na variância fenotípica total foram inferiores a $5 \%$.

Posteriormente, análises bicaráter foram executadas com o objetivo principal de estimar as covariâncias e correlações genéticas entre as características estudadas. Desse modo, foram combinadas duas a duas, assumindo as covariâncias entre os efeitos maternal e direto como zero.

Em termos matriciais, o modelo geral utilizado pode ser descrito como:

$$
y=X \mathrm{~b}+Z_{1} a+Z_{2} m+Z_{3} p+\mathrm{e}
$$

em que: $y=$ vetor das observações da característica; $X=$ matriz de incidência dos efeitos fixos; $\mathrm{b}=$ vetor dos efeitos fixos; $Z_{1}=$ matriz de incidência do efeito genético direto; $a=$ vetor de efeitos genéticos diretos aleatórios; $Z_{2}=$ matriz de incidência do efeito genético materno; $m=$ vetor de efeitos genéticos maternos aleatórios; $Z_{3}=$ matriz de incidência do efeito de ambiente permanente; $p=$ vetor de efeitos aleatórios de ambiente permanente; $\mathrm{e}=$ vetor de efeitos residuais aleatórios.

As pressuposições acerca das distribuições de $y$, $a, m, p$ e e podem ser descritas como:

$$
\left[\begin{array}{l}
y \\
a \\
m \\
p \\
\varepsilon
\end{array}\right] \approx N M V\left\{\left[\begin{array}{l}
X \beta \\
0 \\
0 \\
0 \\
0
\end{array}\right] ; \quad\left[\begin{array}{ccccc}
V & Z_{1} G+Z_{2} C & Z_{1} C+Z_{2} M & Z_{3} P & P \\
G Z_{1}++C Z_{2} & G & C & 0 & 0 \\
C Z_{1}+M Z_{2} & C & M & 0 & 0 \\
P Z_{3} & 0 & 0 & P & 0 \\
R & 0 & 0 & 0 & R
\end{array}\right]\right\}
$$

em que

$$
V=Z_{1} G_{1}{ }^{\prime}+Z_{2} M Z_{2}{ }^{\prime}+2 Z_{1} C Z_{2}{ }^{\prime}+Z_{3} P Z_{3}{ }^{\prime}+R
$$

$G=\mathrm{A} \sigma_{\mathrm{a}}^{2} \mathrm{em}$ análise unicaráter e $\mathrm{A} \oplus \mathrm{G}_{0} \mathrm{em}$ análise bicaráter; $C=\mathrm{A} \sigma_{\mathrm{am}}$ em análise unicaráter e $\mathrm{A} \oplus \mathrm{C}_{0} \mathrm{em}$ 
análise bicaráter; $M=\mathrm{A} \sigma_{\mathrm{m}}{ }^{2} \mathrm{em}$ análise unicaráter e A $\oplus \mathrm{M}_{0} \mathrm{em}$ análise bicaráter; $P=\operatorname{Im} \sigma_{\mathrm{c}}{ }^{2}$ em análise unicaráter e $\mathrm{Im} \AA \mathrm{P}_{0} \mathrm{em}$ análise bicaráter; $R=\operatorname{In} \sigma_{\mathrm{e}}{ }^{2} \mathrm{em}$ análise unicaráter e In $\oplus \mathrm{R}_{0} \mathrm{em}$ análise bicaráter; $m=$ número total de mães; $n=$ número total de observações; $N M V=$ multivariada normal.

Nestas designações, $\sigma_{\mathrm{a}}^{2}$ é a variância genética aditiva direta; $\sigma_{a m}$, a covariância genética entre os efeitos direto e materno; $\sigma_{\mathrm{m}}{ }^{2}$, a variância genética materna; $\sigma_{c}^{2}$, a variância de ambiente materno permanente; $\sigma_{\mathrm{e}}^{2}$, a variância do erro; $\mathrm{G}_{0}, \mathrm{M}_{0}, \mathrm{P}_{0} \mathrm{e} \mathrm{R}_{0}$, as matrizes de (co)variâncias genética direta, genética materna, de ambiente permanente e residual, respectivamente; $\mathrm{C}_{0}$, uma matriz de covariâncias dos efeitos genéticos direto e materno. Assume-se que $\mathrm{P}$ ou $\mathrm{P}_{0}$ e $\mathrm{R}$ ou $\mathrm{R}_{0}$ não são correlacionados com os demais efeitos; A é a matriz de relações aditivas entre os animais; I, uma matriz identidade $\mathrm{e} \oplus$, o produto direto entre as matrizes.

\section{Resultados e Discussão}

O produto de sistemas de bovinos de corte é o crescimento, sendo o objetivo da pecuária de corte a otimização do crescimento pré e pós-natal (Lanna, 1997). Dessa forma, o objetivo da pesquisa nesta área é estudar o crescimento dos animais através dos pesos obtidos a diferentes idades.

Na Tabela 2 são apresentadas as médias, os desviospadrão, os valores máximos e mínimos e o coeficiente de variação de cada característica estudada.

A característica $\mathrm{P} 120$ está presente neste estudo por ser estabelecer ligação com as outras, já que neste período o bezerro sofre pouca influência de manejo e grande influência materna.

As médias de P120 e P550 estão de acordo com as encontradas na literatura consultada para a raça Nelore (Salles, 1995; Lôbo et al., 1997). No entanto, o valor médio encontrado para a característica P550 é superior ao valor citado, também na raça Nelore no Estado do Ceará, por Martins Filho et al. (1997) que provavelmente foi influenciado pelas condições ambientais.

O valor médio encontrado na literatura (Sumário de Touros Nelore 2001) é de $280,8 \mathrm{~kg}$ para peso aos 450 dias, de 59.353 observações das fazendas da Agro-Pecuária CFM, sendo superior ao valor encontrado no presente estudo P455 (250kg). Estes dados não foram previamente ajustados para idade, pois estas são incluídas no modelo matemático que se encarrega de efetuar o ajuste.
Tabela 2 - Média geral, desvio-padrão (DP), coeficiente de variação (CV), valores máximos (Max.) e mínimos (Min.) das características estudadas $(\mathrm{kg})$

Table 2 - Means, standard deviation (DP), coefficient of variation (CV), minimum (Min) and maximum (Max) values for studied characteristics $(\mathrm{kg})$

\begin{tabular}{lccccc}
\hline $\begin{array}{l}\text { Características } \\
\text { Characteristics }\end{array}$ & $\begin{array}{c}\text { Média } \\
\text { Mean }\end{array}$ & DP & Min. & Max. & CV $(\%)$ \\
\hline P120 & 119 & 18 & 48 & 202 & 15 \\
P455 & 250 & 44 & 106 & 491 & 18 \\
P550 & 293 & 49 & 140 & 574 & 17 \\
\hline
\end{tabular}

$\mathrm{P} 120=$ peso padronizado aos 120 dias de idade; $\mathrm{P} 455=$ peso padronizado aos 455 dias de idade; $\mathrm{P} 550=$ peso padronizado aos 550 dias de idade.

Em análises mais recentes realizadas por Lôbo et al. (2001), incluindo 147 rebanhos participantes do PMGRN, com 74.215 registros para peso aos 450 dias, o valor médio encontrado foi de $290 \mathrm{~kg}$.

Há tendência desses valores fenótipicos aumentarem anualmente, já que os criadores que participam de um programa de melhoramento são orientados a utilizarem nos acasalamentos das matrizes, touros que possuem valores genéticos superiores a média do seu rebanho.

Os efeitos ambientais, como ano e época de nascimento, fazenda, onde estão incluídos o clima, solo, índice pluviométrico, manejo etc, são importantes na determinação de uma característica, e são denominados de efeitos fixos em uma avaliação genética.

A correta definição dos grupos contemporâneos é um dos aspectos mais importantes de qualquer programa de melhoramento genético e avaliação genética, pois parte dos efeitos fixos é considerada nos grupos.

As estimativas dos componentes de variância e herdabilidades obtidas nas análises unicarater são apresentadas na Tabela 3.

Para a característica P120, observou-se estimativa do coeficiente de herdabilidade direta de 0,29, que é superior as estimativas encontradas na literatura consultada para a raça Nelore (Lôbo et al., 1997; Reyes et al., 1997; Garnero et al., 2001). Os últimos autores utilizaram o mesmo modelo usado neste estudo em análise unicarater, empregando-se também o MTDFREML. No entanto, o valor do coeficiente de herdabilidade materna $(0,08)$ é inferior aos valores relatados pelos referidos autores.

O coeficiente de herdabilidade direta estimado para a característica P550, ou seja, 0,47, é um valor alto, 
Tabela 3 - Estimativas dos componentes de variância e herdabilidades, segundo análise unicaráter para pesos padronizados aos 120 (P120), aos 455 (P455) e aos 550 (P550) dias em bovinos da raça Nelore

Table 3 - Estimates of the variance components and heritability with one-trait analysis for standardized weight at 120 (P120), at 455(P455) and at 550 (P550) days in Nellore cattle

\begin{tabular}{lccrrrrr}
\hline $\begin{array}{l}\text { Características } \\
\text { Characteristics }\end{array}$ & $\sigma^{2}{ }_{\mathrm{a}}$ & $\sigma_{\mathrm{m}}^{2}$ & $\sigma_{\mathrm{pe}}^{2}$ & $\sigma^{2}$ & $\sigma_{\mathrm{e}}^{2}$ & $\mathrm{~h}^{2}{ }_{\mathrm{a}}$ & $\mathrm{h}^{2}{ }_{\mathrm{m}}$ \\
\hline P120 & & & & & & \\
P455 & 49,05 & 12,84 & 24,21 & 83,26 & 169,37 & 0,29 & 0,08 \\
P550 & 379,81 & - & 368,03 & 747,84 & 0,51 & - & - \\
\hline
\end{tabular}

$\sigma_{a}^{2}=$ variância genética direta; $\sigma_{m}^{2}=$ variância genética materna; $\sigma_{p e}^{2}=$ variância de ambiente permanente $\sigma^{2}=$ variância residual; $\sigma_{p}^{2}=$ variância fenotípica; $h_{a}^{2}=$ herdabilidade direta; $h_{m}^{2}=$ herdabilidade materna.

$\sigma_{a}^{2}=$ direct genetic variance; $\sigma_{m}^{2}=$ maternal genetic variance; $\sigma_{p e}^{2}=$ permanent environmental variance; $\sigma_{e}^{2}=$ residual variance; $\sigma_{p}^{2}=$ phenotypic variance; $h^{2}{ }_{a}=$ direct heritability; $h_{m}^{2}=$ maternal heritability.

sendo superior às estimativas citadas na literatura consultada (Eler et al., 1995; Reyes et al., 1997; Garnero et al., 2001), que variaram de 0,16 a 0,31 .

A estimativa de 0,51 , obtida para o coeficiente de herdabilidade direta de P455, foi maior que as estimadas para os outros pesos. Todavia, verificaram-se na literatura (Mercadante,1995) valores de estimativas de herdabilidade que variam de 0,03 a 0,80 para $\mathrm{P} 450$, um artigo de revisão sobre parâmetros genéticos para características de crescimento em zebuínos de carne.

Nas Tabelas 4 e 5 são apresentadas as estimativas dos parâmetros genéticos obtidos para as características estudadas, mediante análises bicaráter.

Os coeficientes de herdabilidade direta obtidos para P120 (0,58 e 0,53), são superiores aos estimados por Lôbo et al. (1997) e Reyes et al. (1997). No entanto, as estimativas da herdabilidade materna $(0,02$ e 0,03$)$ foram inferiores. Para a característica P550, as estimativas de herdabilidade direta $(0,44 \mathrm{e}$ 0,49 ) foram de magnitude maior que as estimadas por Eler et al. (1995) e Reyes et al. (1997).

Verifica-se também, na Tabela 5, que as estimativas dos coeficientes de herdabilidade direta para P455 $(0,53$ e 0,50$)$, tal como as estimadas para P120 e P550, foram altas, indicando que grande parte da variância fenotípica das características estudadas pode ser atribuída aos efeitos aditivos dos genes. Por outro lado, as estimativas das correlações genéticas de P120 x P455, P120 x P550 e P455 x P550 (0,92, 0,93 e 0,96, respectivamente), sugerem que existe uma associação linear positiva entre as características, sendo que grande parte dos genes que determinam uma característica, determina, também, as demais.

Os valores encontrados no presente estudo para as variâncias genética direta e herdabilidade direta para P455 e P550, tanto em análise unicarater como na bicarater, são substancialmente altos, isto provavelmente se deva ao fato do modelo matemático adotado para análise destas duas características não incluirem o efeito de ambiente permanente da vaca.

Mercadante (1997), fez um estudo com o objetivo de apresentar estimativas dos componentes de (co)variância e dos parâmetros genéticos para algumas características de crescimento de fêmeas de um rebanho da raça Nelore, no intuito de quantificar a importância dos efeitos genéticos direto e materno e efeito de ambiente permanente. Nota-se, no estudo em que se avaliaram quatro modelos diferentes, que a não inclusão dos efeitos genético materno e de ambiente permanente, pode ocasionar mudanças significativas nas estimativas dos componentes e das herdabilidades, podendo até levar à superestimação destes valores e, conseqüentemente, o valor genético dos animais para estas características. A autora trabalhou com apenas duas características, peso aos 240 e aos 365 dias.

Portanto, o objetivo do nosso estudo foi estimar componentes e parâmetros genéticos não com o intuito de se estimar os valores genéticos dos animais, e sim analisar a viabilidade de utilizar o peso padronizado aos 455 dias como critério de seleção em substituição ao peso padronizado aos 550 dias no PMGRN.

Sabemos que o aumento da eficiência do setor cria, que é responsável pela maior parte do sistema de bovinos de corte, pode ser atingido pela redução na idade ao primeiro parto, sendo alcançada quando elevada proporção de novilhas apresenta cio ao início da estação de monta, aos 15 meses de idade.

\footnotetext{
R. Bras. Zootec., v.32, n.1, p.99-105, 2003
} 
Tabela 4 - Estimativas dos componentes de (co)variância, segundo análise bicaráter para pesos padronizados aos 120 (P120), 455 (P455) e 550 (P550) dias de idade para bovinos da raça Nelore

Table 4 - Estimates of the (co)variance components with two-traits analysis for standardized weight at $120(P 120)$, at $455(P 455)$ and at 550 (P550) days in Nellore cattle

\begin{tabular}{|c|c|c|c|}
\hline \multirow[t]{2}{*}{$\begin{array}{l}\text { Componentes } \\
\text { Component }\end{array}$} & \multicolumn{3}{|c|}{$\begin{array}{c}\text { Características } \\
\text { Characteristic }\end{array}$} \\
\hline & (1)P120x(2)P455 & (1)P120x(2)P550 & (1)P455 x(2)P550 \\
\hline 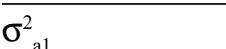 & 105,84 & 82,69 & 374,81 \\
\hline$\sigma^{2}$ & 398,51 & 446,10 & 461,11 \\
\hline & 189,79 & 178,82 & 398,83 \\
\hline$-{ }^{2}$ & 4,45 & 4,89 & - \\
\hline 1 & 9,91 & 10,51 & - \\
\hline el & 63,25 & 67,63 & 373,11 \\
\hline$\sigma^{2}$ & 356,18 & 559,25 & 486,94 \\
\hline 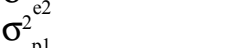 & 183,44 & 165,73 & 347,92 \\
\hline$\sigma^{2}$ & 754,69 & 1005,35 & 948,06 \\
\hline
\end{tabular}

$\sigma^{2}=$ variância genética direta; $\sigma^{2}=$ variância genética materna; $\sigma^{2}=$ variância de ambiente permanente; $\sigma^{2}=$ variância fenotípica; $\sigma^{2}{ }^{2 \mathrm{ii}}=$ variância residual da característica $\mathrm{i}(\mathrm{i}=1,2) ; \sigma_{\mathrm{a12} 2}=$ covariância genética direta entre as características 1 e 2 .

$\sigma_{a i}^{2}=$ direct genetic variance; $\sigma_{m i}^{2}=$ maternal genetic variance; $\sigma_{p e i}^{2}=$ permanent environmental variance; $\sigma_{p i}^{2}=$ phenotypic variance; $\sigma_{e i}^{2}=$ residual variance for the characteristic $i\left(i=1\right.$ e 2); and $\sigma_{a 122}=$ direct genetic covariance among the characteristics 1 and 2 .

Tabela 5 - Coeficientes de herdabilidade e de correlação genética, segundo análise bicaráter para os pesos padronizados aos 120(P120), aos 455(P455) e aos 550(P550) dias de idade para bovinos da raça Nelore

Table 5 - Estimates of the heritabilities and genetic correlation in two-traits analysis for standardized weight at 120(P120), 455(P455) and 550(P550) days in Nellore cattle

\begin{tabular}{lcccc}
\hline $\begin{array}{l}\text { Características } \\
\text { Characteristics }\end{array}$ & $\mathrm{h}^{2}{ }_{\mathrm{a} 1}$ & $\mathrm{~h}^{2}{ }_{\mathrm{a} 2}$ & $\mathrm{~h}_{\mathrm{m} 1}$ & $\mathrm{r}_{\mathrm{a} 1 \mathrm{a} 2}$ \\
\hline (1)P120x(2)P455 & 0,58 & 0,53 & 0,02 & 0,92 \\
(1)P120x(2)P550 & 0,50 & 0,44 & 0,03 & 0,93 \\
(1)P455x(2)P550 & 0,50 & 0,49 & - & 0,96 \\
\hline
\end{tabular}

$\mathrm{h}^{2}{ }_{11}=$ herdabilidade direta da característica $1 ; \mathrm{h}^{2}{ }_{22}=$ herdabilidade direta da característica $2 ; \mathrm{h}^{2}{ }_{\mathrm{m} 1}=$ herdabilidade materna da característica $1 ; r_{112}=$ correlação genética entre as características 1 e 2 .

$h^{2}{ }_{a 1}$ direct heritability for the characteristic $1 ; h^{2}$ direct heritability for the characteristic $2 ; h^{2}=$ maternal heritability for the characteristic $1 ; r_{a 1 a 2}=$ genetic correlation among the characteristics 1 and 2.

A aceleração da maturidade sexual pode ser obtida através da utilização de uma série de técnicas de manejo, mas é ultimamente limitada pelo potencial genético (Lanna, 1997). Assim, estimular e orientar os criadores a selecionar os animais à idades mais jovens, e desperta-los para outras características de interesse econômico, além dos pesos, é de grande responsabilidade dos Programas de Melhoramento Genético, já que os reprodutores produzidos nestes rebanhos servirão a base da pirâmide, que são os rebanhos comerciais.

\section{Conclusões}

A característica $\mathrm{P} 455$ possui variabilidade genética aditiva de magnitude tal, que permite ser modificada por seleção. Isto, aliado ao fato de serem altas as estimativas das correlações genéticas observadas entre P455 e as demais características, sugere que o peso aos 455 dias de idade pode ser adotado como critério de seleção. No entanto, novos estudos são necessários para investigar possíveis associações do $\mathrm{P} 455$ com outras características de importância econômica. 
A característica P120, por ser pouca influenciada pelo manejo, é a utilizada para se avaliar a habilidade maternal da mãe e de crescimento pré-desmama do bezerro, sendo de fundamental importância para o PMGRN, pois é usada como âncora nas análises bicaracteres para minimizar os efeitos de descartes posteriores à desmama.

Para a estimativa do P550, os animais devem ser pesados pelo menos mais uma vez, fazendo com que permaneçam no rebanho por pelo menos três meses a mais. Dessa forma, a alta correlação genética com o P455 permite a redução do número de pesagens e a utilização do P455 como critério de seleção.

\section{Agradecimento}

Ao Programa de Melhoramento Genético da Raça Nelore (PMGRN), dos criadores participantes e do GEMAC, pelo apoio.

\section{Literatura Citada}

BOLDMAN, K.G.; KRIESE, L.A.; Van VLECK, L.D. et al. A manual for use for MTDFREML. A set of programs to obtain of variance and covariances [DRAF]. Lincoln: Agricultural Research Service, 1995. 120p.

CFM. Sumário de Touros Nelore 2001. Publicação da AgroPecuária CFM. São José do Rio Preto: Agro-Pecuária CFM, 2001, 60p.

ELER, J.P.; Van VLECK, L.D.; FERRAZ, J.B.S. et al. Estimation of variances due to direct and maternal effects for growth traits of Nelore cattle. Journal of Animal Science, v.77, p.3253-3258, 1995.

GARNERO, A. del V.; LÔBO, R.B.; BEZERRA, L.A.F. et al. Comparação entre alguns critérios de seleção para Crescimento na Raça Nelore. Revista Brasileira de Zootecnia, v.30, n.3, p.714-718, 2001.
LANNA, D.P. Fatores condicionantes e predisponentes da puberdade e da idade de abate. In: SIMPÓSIO SOBRE PECUÁRIA DE CORTE, 4., 1996, Piracicaba. Anais...Piracicaba, 1997. p.41-78.

LÔBO, R.B.; REYES, A. de los; BEZERRA, L.A.F. et al. Avaliação genética de animais jovens, touros e matrizes. Ribeirão Preto: GEMAC - Departamento de Genética/Universidade de São Paulo, 1997. 65p.

LÔBO, R.B.; BEZERRA, L.A.F.; OLIVEIRA, H.N. et al. Avaliação genética de animais jovens, touros e matrizes. Ribeirão Preto: GEMAC - Departamento de Genética/Universidade de São Paulo, 2001.60p.

MARTINS FILHO, R.; LOBO, R.N.B.; LIMA, F.A.M. et al. Parâmetros genéticos e fenotípicos de pesos e ganhos em pesos de bovinos Zebus no estado do Ceará. In: REUNIÃO ANUAL DA SOCIEDADE BRASILEIRA DE ZOOTECNIA, 34, 1997, Juiz de Fora. Anais... Juiz de Fora, 1997. 600p. p.248-250.

MERCADANTE, M.E.Z., LÔBO, R.B Estimativas de (co)variâncias e parâmetros genéticos dos efeitos direto e materno de características de crescimento de fêmeas de um rebanho Nelore. Revista Brasileira de Zootecnia, v.26, n.6, p.1124-1133, 1997.

REYES, A. de los; LÔBO, R.B.; BEZERRA, L.A.F. et al. Variabilidade genética de características de crescimento alternativas para a seleção em gado de corte. In: REUNIÃO ANUAL DA SOCIEDADE BRASILEIRA DE ZOOTECNIA, 34, 1997, Juiz de Fora. Anais... Juiz de Fora, 1997. 600p. p.245-247.

SALLES, P.A. Critérios de seleção para características de crescimento em machos da raça Nelore. Ribeirão Preto: Universidade de São Paulo, 1995. 65p. Dissertação (Mestrado em Genética) - Universidade de São Paulo, 1995.

SAS INSTITUTE. SAS user's guide for windows enviroment. 6.08. Cary: 1995.

Recebido em: 19/06/01

Aceito em: 22/08/02 